CLINICAL STUDY

\title{
The prognostic value of the metastatic lymph node ratio and maximal metastatic tumor size in pathological N1 a papillary thyroid carcinoma
}

\author{
Min Ji Jeon ${ }^{1}$, Jong Ho Yoon ${ }^{2}$, Ji Min Han $^{1}$, Ji Hye Yim ${ }^{1}$, Suck Joon Hong ${ }^{2}$, Dong Eun Song ${ }^{3}$, Jin-Sook Ryu ${ }^{4}$, \\ Tae Yong Kim ${ }^{1}$, Young Kee Shong ${ }^{1}$ and Won Bae Kim ${ }^{1}$ \\ Departments of ${ }^{1}$ Internal Medicine, ${ }^{2}$ Surgery, ${ }^{3}$ Pathology and ${ }^{4}$ Nuclear Medicine, Asan Medical Center, University of Ulsan College of Medicine, \\ 388-1 Pungnap-dong, Songpa-gu, Seoul 138-736, Republic of Korea
}

(Correspondence should be addressed to W B Kim; Email: kimwb@amc.seoul.kr)

\begin{abstract}
Objective: The presence of central neck lymph node (LN) metastases (defined as pN1a according to Tumor Node Metastasis classification) in papillary thyroid cancer (PTC) is known as an independent risk factor for recurrence. Extent of LN metastasis and the completeness of removal of metastatic LN must have an impact on prognosis but they are not easy to measure. Moreover, the significance of the size of metastatic tumors in LNs has not been clarified. This study was to evaluate the impact of the extent of LN metastasis and size of metastatic tumors on the recurrence in pathological N1a PTC. Design: This retrospective observational cohort study enrolled 292 PTC patients who underwent total thyroidectomy with central neck dissection from 1999 to 2005. LN ratio was defined as the number of metastatic LNs divided by the number of removed LNs, which was regarded as variable reflecting both extent of LN metastasis and completeness of resection, and LN size as the maximal diameter of tumor in metastatic LN.

Results: The significant risk factors for recurrence in univariate analysis were large primary tumor size (defined as larger than $2 \mathrm{~cm}$ ), high LN ratio (defined as higher than 0.4), and presence of macrometastasis (defined as larger than $0.2 \mathrm{~cm}$ ). Age, sex, clinical node status, and microscopic perithyroidal extension had no effect on recurrence. In multivariate analysis, high LN ratio and presence of macrometastasis were independent risk factors for recurrence.

Conclusion: LN ratio and size of metastatic nodes had a significant prognostic value in pathological N1a PTC. We suggest that risk stratification of pathological N1a PTC according to the pattern of LN metastasis such as LN ratio and size would give valuable information to clinicians.
\end{abstract}

European Journal of Endocrinology 168 219-225

\section{Introduction}

Regional neck lymph node (LN) metastases are found in up to $70 \%$ of cases of papillary thyroid carcinoma (PTC) and the presence of neck LN metastases has been identified as an independent risk factor for recurrence $(1,2,3,4)$. American Joint Committee on Cancer (AJCC) classification system based on pathological tumor node metastasis (TNM) parameters and age categorizes nodal status into N1a and N1b according to the compartment of neck nodes but the prognosis of PTC patients with pathological N1a are quite variable (3). It is difficult to clarify the prognostic factors and to find optimal management strategies for PTCs because of its indolent clinical course (4).

LN ratio after lymphadenectomy has been known as a prognostic variable in nonthyroidal cancers such as gastric, breast, and colorectal cancers. Complete surgical removal of the involved LN may lead to clearly better prognosis even with the large number of involved LNs $(5,6,7)$. In breast cancers, LN size is also an important prognostic factor. The term 'micrometastases' was arbitrarily defined in the breast cancer staging system in 1971 on the basis of $0.2 \mathrm{~cm}$ and it subclassifies N1 disease $(8,9)$.

Several studies also suggested that larger size or higher number of metastatic LNs in PTC is associated with poorer outcomes $(1,10,11,12,13)$. A recent review suggested new risk factors that modify standard N1 risk of recurrence such as clinical status, size, number, or presence of extranodal extension of metastatic lesions in LNs (14).

Here, we evaluated the prognostic value of metastatic LN ratio and maximal metastatic tumor size 
in pathological N1a PTC patients who underwent operation in a single tertiary referral hospital with postoperative radioactive iodine (RAI) ablation therapy.

\section{Materials and methods}

\section{Patients}

Between 1999 and 2005, 602 conventional PTC patients who underwent total thyroidectomy, routine central neck dissection by a single surgeon $(\mathrm{S} \mathrm{J} \mathrm{H})$, and subsequent RAI ablation therapy according to a protocol established by the Endocrinology Division of the Asan Medical Center (Seoul, Korea) were retrieved (15). The median dose of administered radioiodine was $5.6 \mathrm{GBq}$. Among them, we only included patients with four or more removed central LNs and with the maximal diameter of primary tumor $>1 \mathrm{~cm}$. Patients with lateral neck $\mathrm{LN}$ involvement or distant metastasis, gross invasion, or resection margin involvement by tumor were excluded. Finally, 292 patients were eligible for analysis (Fig. 1). This study was approved by the institutional review board.

\section{Definitions}

Clinical node status was defined as follows: clinical N1 (cN1) was defined as the presence of suspicious central neck LNs identified by preoperative physical examination, preoperative imaging and/or gross inspection

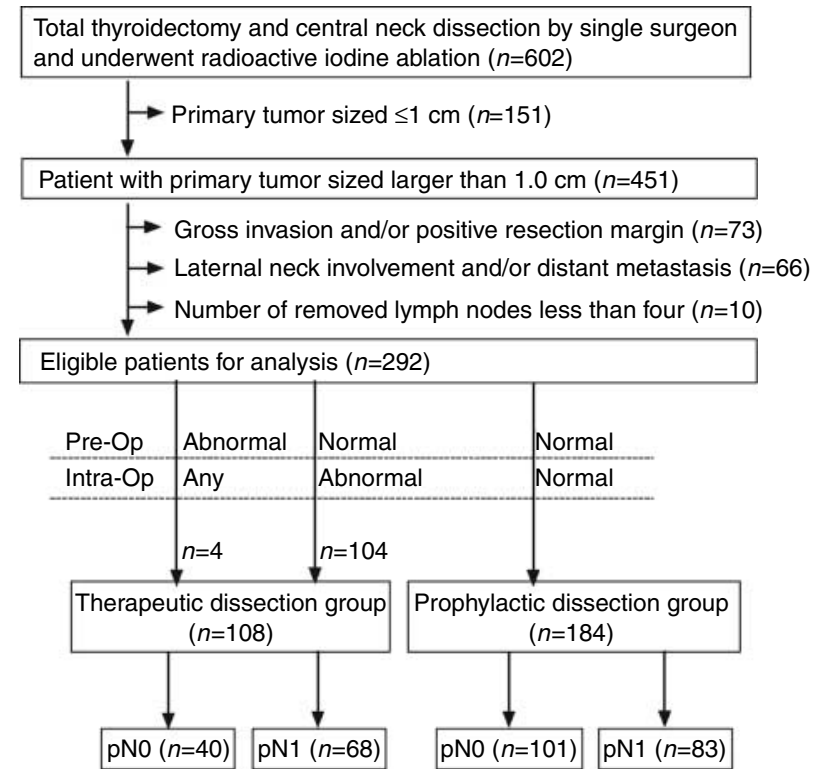

Figure 1 Description of study cohort. This algorithm demonstrated how the final enrolled patients were retrieved. A total of 292 patients were classified into therapeutic dissection group and prophylactic dissection group according to preoperative physical examination, ultrasonograpy, and surgical findings. during surgery. Clinical NO (cNO) was defined as the absence of suspicion for LN involvement.

All the patients eligible for analysis received central neck node dissection (by the inclusion criteria regarding the number of removed nodes). We divided patients into two groups according to clinical node status. Thus, a group of patients with $\mathrm{cN} 1$ was defined as 'therapeutic dissection group', and the other group of patients with cNO was defined as 'prophylactic dissection group'. Pathological N1a (pN1a) was defined as the presence of metastatic LN among four or more removed LNs from central neck and pathological NO (pNO) was defined as the absence of metastasis in pathological examination.

$\mathrm{LN}$ ratio was defined as the number of metastatic LNs divided by the number of removed LNs. It represented the extent of LN involvement and completeness of LN dissection. Receiver Operating Characteristic curve (ROC) curve analysis showed that 0.38 is the appropriate cutoff point of LN ratio (data are not shown). So, we defined low $\mathrm{LN}$ ratio as $\leq 0.4$ and high $\mathrm{LN}$ ratio as $>0.4$.

LN size, which means the maximal tumor diameter in the metastatic LNs, was classified into two groups on the basis of the largest LN among metastatic LN using the concept of micrometastasis in breast cancer $(8,9,16)$ : micrometastasis, $\leq 0.2 \mathrm{~cm}$; macrometastasis, $>0.2 \mathrm{~cm}$.

The patients were classified into three groups by the $\mathrm{LN}$ ratio and $\mathrm{LN}$ size: Low, low $\mathrm{LN}$ ratio with micrometastasis; Intermediate, low LN ratio with macrometastasis or high LN ratio with micrometastasis; and High, high LN ratio with macrometastasis.

\section{Pathological examination}

The entire specimen from the operation was submitted for histological analysis to find LN metastasis and the maximal tumor diameter in the metastatic LN was recorded. For this study, an experienced pathologist (D E S) reviewed the pathological slides and measured the size of metastatic foci in each LN.

\section{Follow-up protocol to detect recurrence}

After remnant ablation, all patients were regularly followed with physical examination and serum $\mathrm{Tg}$ measurement every 6-12 months. Neck ultrasonographic examinations were done in all patients during the entire follow-up periods at 6- to 12-month intervals. Additional diagnostic imaging studies, such as diagnostic radioiodine whole-body scan, computed tomography, magnetic resonance scan, or whole-body fluorodeoxyglucose-positron emission tomography scan, were also performed in some patients. Clinical recurrence was defined as the structural disease recurrence; reappearance of pathologically proven malignant tissue and/or appearance of metastatic lesions in other organs by imaging studies. 
Table 1 Univariate analysis of risk factors of recurrence.

\begin{tabular}{|c|c|c|c|}
\hline Classification & $n(\%)$ & Hazard ratio $(95 \% \mathrm{Cl})$ & $P$ value \\
\hline \multicolumn{4}{|l|}{ Age (years) } \\
\hline$<45$ & $150(51)$ & Ref & \\
\hline$\geq 45$ & 142 (49) & 0.75 (0.39 to 1.45$)$ & 0.393 \\
\hline \multicolumn{4}{|l|}{ Sex } \\
\hline Male & $28(10)$ & Ref & \\
\hline Female & $264(90)$ & $0.60(0.23$ to 1.53$)$ & 0.283 \\
\hline \multicolumn{4}{|l|}{ Tumor size $(\mathrm{cm})$} \\
\hline$\leq 2.0$ & $180(62)$ & Ref & \\
\hline$>2.0$ & $112(39)$ & 2.71 (1.38 to 5.29$)$ & 0.004 \\
\hline \multicolumn{4}{|c|}{ Perithyroidal extension } \\
\hline Absent & $116(40)$ & Ref & \\
\hline Present & $176(60)$ & 1.23 (0.62 to 2.46$)$ & 0.558 \\
\hline \multicolumn{4}{|l|}{ Clinical status } \\
\hline $\mathrm{cNO}$ & $184(63)$ & Ref & \\
\hline $\mathrm{cN} 1$ & $108(37)$ & $1.84(0.96$ to 3.54$)$ & 0.068 \\
\hline \multicolumn{4}{|l|}{ Metastasis to LN } \\
\hline pNO & $141(48)$ & $\operatorname{Ref}^{a}$ & \\
\hline pN1a & $151(52)$ & 8.22 (2.91 to 23.25 ) & $<0.001$ \\
\hline \multicolumn{4}{|l|}{ LN ratio } \\
\hline $\operatorname{Low}(\leq 0.4)$ & $89(59)$ & $3.22(0.97$ to 10.71$)$ & 0.056 \\
\hline High $(>0.4)$ & $62(41)$ & $17.14(5.94$ to 49.46$)$ & $<0.001$ \\
\hline \multicolumn{4}{|l|}{ LN size } \\
\hline $\begin{array}{l}\text { Micrometastasis } \\
(\leq 0.2 \mathrm{~cm})\end{array}$ & $59(39)$ & 4.12 (1.21 to 14.08$)$ & 0.024 \\
\hline $\begin{array}{l}\text { Macrometastasis } \\
(>0.2 \mathrm{~cm})\end{array}$ & $92(61)$ & 11.42 (3.97 to 32.83$)$ & $<0.001$ \\
\hline \multicolumn{4}{|l|}{ Patient's group } \\
\hline Low & $46(31)$ & $2.23(0.50$ to 9.95$)$ & 0.295 \\
\hline Intermediate & $56(37)$ & $6.07(1.87$ to 19.70$)$ & 0.003 \\
\hline High & 49 (32) & $19.08(6.51$ to 55.93$)$ & $<0.001$ \\
\hline
\end{tabular}

$\mathrm{Cl}$, confidence interval; Ref, reference; $\mathrm{LN}$ ratio, metastatic $\mathrm{LNs} /$ removed LNs; patient group according to the ratio and size of LN.

${ }^{a} \mathrm{pNO}$ is a reference group to compare risk of recurrence in $L N$ ratio, $L N$ size, and patient group.

\section{Statistical analysis}

Statistics were calculated by $\mathrm{R}$ version 2.13 and $\mathrm{R}$ libraries survival, car and Cairo were used to analyze the data and draw graphs (R Foundation for Statistical Computing, Vienna, Austria, http://www.R-project. org). We also used SPSS software (version 18.0.1; SPSS, Inc.) for ROC curve analysis. Continuous variables were presented as medians with interquartile range (IQR) and categorical variables were presented as numbers with percentages. Cox proportional hazard model was used to evaluate the risk of recurrence. Univariate analyses were performed on age, sex, tumor size, microscopic extrathyroidal extension (ETE), clinical node status, LN size, LN ratio, and patient group. Multivariate analyses included the variables that were significant in the univariate analysis. Because LN size, LN ratio, and patient group were closely related, they were separately analyzed in multivariate analysis. Recurrence-free survival curves were constructed using Kaplan-Meier method and log rank tests were used to evaluate differences of recurrence-free survival between patient groups. We also analyzed the impact of these variables on recurrence in each dissection group. All $P$ values were two sided, with $P<0.05$ considered statistically significant.

\section{Results}

\section{Baseline characteristics of patients and LNs}

A total of 292 patients were evaluated in this study, and they were followed for a median of 8 years (IQR 5.8-10.0). Median age of the patients was 44.4 years (IQR 35.1-54.2) and 264 (90\%) patients were female. Median primary tumor size was $1.8 \mathrm{~cm}$ (IQR $1.5-2.5)$. Of 292 patients, 108 (37\%) were therapeutic dissection group and $184(63 \%)$ were prophylactic dissection group. Among 108 patients in the therapeutic dissection group, four had enlarged LNs in preoperative physical examination or preoperative ultrasonography and 104 in intra-operative gross inspection (Fig. 1). Using TNM system (a classification system of the AJCC, 6th ed., 2002), 172 (59\%) patients were stage I, seven $(2 \%)$ were stage II, and $113(39 \%)$ were stage III.

The median number of resected LN per patient was 9 (IQR 6-13). Of the 292 patients, 151 (52\%) showed metastases to central neck and the median number of metastatic LN was 3 (IQR 2-5.5). Patients with high LN ratio were $62(41 \%)$ and those with low LN ratio were 89 (59\%). The mean metastatic LN size was $0.3 \mathrm{~cm}$ (IQR 0.2-0.5). Among patients with LN metastasis, 92 (61\%) showed macrometastasis and 59 (39\%) showed micrometastasis. Group Low included 46 (31\%), group Intermediate included $56(37 \%)$, and group High included $49(32 \%)$ patients (Table 1$)$.

Thirty-six (12\%) events of recurrence had occurred during the follow-up period. Thirty-three $(92 \%)$ of them were lateral neck recurrences, two were recurrences at operation bed, and one was metastasis to mediastinal LN. Most of the recurrences $(34,94 \%)$ were treated by

Table 2 Multivariate analysis of risk factors of recurrence.

\begin{tabular}{|c|c|c|c|}
\hline & Classification & Hazard ratio $(95 \% \mathrm{Cl})$ & $P$ value \\
\hline \multicolumn{4}{|l|}{ Model A } \\
\hline Tumor size & $>2.0 \mathrm{~cm}$ & $1.82(0.93$ to 3.60$)$ & 0.083 \\
\hline \multirow[t]{2}{*}{ LN ratio } & Low $(\leq 0.4)$ & $2.92(0.88$ to 9.76$)$ & 0.081 \\
\hline & High & $14.83(5.07$ to 43.35$)$ & $<0.001$ \\
\hline \multicolumn{4}{|l|}{ Model B } \\
\hline Tumor size & $>2.0 \mathrm{~cm}$ & 1.91 (0.97 to 3.77$)$ & 0.061 \\
\hline \multirow[t]{2}{*}{ LN size } & $\begin{array}{l}\text { Micrometastasis } \\
(\leq 0.2 \mathrm{~cm})\end{array}$ & 3.74 (1.09 to 12.84$)$ & 0.036 \\
\hline & $\begin{array}{l}\text { Macrometastasis } \\
\quad(>0.2 \mathrm{~cm})\end{array}$ & 9.86 (3.39 to 28.68 ) & $<0.001$ \\
\hline \multicolumn{4}{|l|}{ Model C } \\
\hline Tumor size & $>2.0 \mathrm{~cm}$ & $1.82(0.9$ & 0.085 \\
\hline Patient & Low & 2.07 (0.46 to 9.28$)$ & 0.341 \\
\hline \multirow{2}{*}{ group } & Intermediate & 5.31 (1.62 to 17.43$)$ & 0.006 \\
\hline & High & 16.55 (5.58 to 49.11$)$ & $<0.001$ \\
\hline
\end{tabular}

Model A analyzed tumor size and LN ratio; Model B analyzed tumor size and LN size; Model $C$ analyzed tumor size and patient group; LN ratio, metastatic LNs/removed LNs; Patient group according to the ratio and size of LN. 


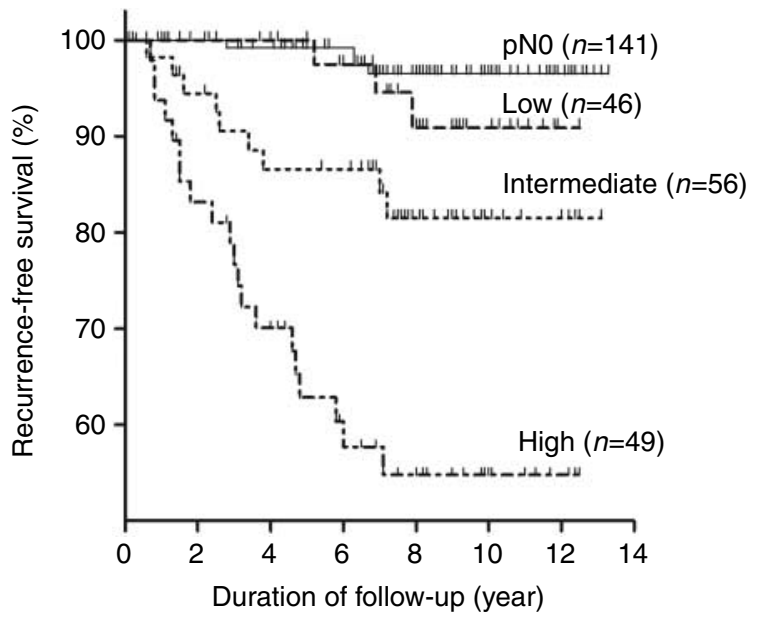

Figure 2 Recurrence-free survival according to the patient groups classified by both LN ratio and size: Low, low LN ratio with micrometastasis; Intermediate, low $L N$ ratio with macrometastasis or high $\mathrm{LN}$ ratio with micrometastasis; and High, high $\mathrm{LN}$ ratio with macrometastasis. Recurrence-free survival rate of each group were $96.5 \%$ in pNo, $90.9 \%$ in group Low, $81.5 \%$ in group Intermediate and $54.8 \%$ in group High. There was significant difference in recurrence-free survival among patient groups. (Log rank statistics $=60.9, P<0.001$ ).

surgical excision, two with lateral neck recurrences by RAI, one with lateral neck recurrences by ethanol injection, and one with mediastinal metastasis by radiotherapy. There were three mortality events including one of disease-specific mortality from patients with lateral neck recurrence.

\section{Impact of $L N$ ratio and $L N$ size on recurrence}

In univariate analysis of risk factors for recurrence, primary tumor of $>2 \mathrm{~cm}$ and pN1a compared with pNO were significantly associated with recurrence of PTC. Age, sex, presence of microscopic perithyroidal extension, and clinical node status were not risk factors for recurrence. We subdivided pN1a group on the basis of LN ratio and LN size. High LN ratio showed a higher hazard ratio compared with the $\mathrm{pNO}$ group. Not only macrometastasis but also micrometastasis of LN was associated with higher risk of recurrence than pNO group. In consideration of both LN ratio and LN size, group Low did not show any significant impact on prognosis (Table 1).

Multivariate analysis on tumor size, LN size, LN ratio, and patient's group, which were significant factors associated with recurrences in univariate analysis, revealed that high LN ratio and presence of macrometastasis were independently associated with recurrence. Micrometastasis also carries a risk higher than pathological NO but lower than macrometastatic disease after adjusting for tumor size. Group Low showed higher risk of recurrence when compared with pNO but it was not statistically significant (hazard ratio 2.07, 95\% confidence interval (CI) 0.46 to $9.28, P=0.341$ ) after adjusting for tumor size. This result suggests that LN ratio has a more distinct effect on prognosis than LN size. Patients in group Intermediate and group High showed significantly higher risk of recurrence (hazard ratio 5.31, $95 \% \mathrm{CI} 1.62$ to $17.43, P=0.006$ in Intermediate group; hazard ratio $16.55,95 \%$ CI 5.58 to 49.11 , $P<0.001$ in High group) (Table 2). Kaplan-Meier curves for recurrence-free survival according to the patient groups is shown in Fig. 2, and there was a clear association between prognosis and patient groups $\left(\chi^{2}=60.9, P<0.001\right)$.

\section{Impact of $L N$ ratio and $L N$ size on recurrence in each dissection group}

Of 108 patients in therapeutic dissection group, 40 patients $(37 \%)$ were $\mathrm{pNO}$ even though they had cN1 disease. Higher LN ratio and larger LN size were also significant risk factors in this group. However, the

Table 3 Impact of LN ratio, LN size, and patient group on recurrence in each dissection group.

\begin{tabular}{|c|c|c|c|c|c|c|}
\hline \multirow[b]{2}{*}{ Classification } & \multicolumn{3}{|c|}{ Therapeutic dissection group } & \multicolumn{3}{|c|}{ Prophylactic dissection group } \\
\hline & $n(\%)$ & Hazard ratio $(95 \% \mathrm{Cl})$ & $P$ value & $n(\%)$ & Hazard ratio $(95 \% \mathrm{Cl})$ & $P$ value \\
\hline \multicolumn{7}{|l|}{ Metastasis to LN } \\
\hline pNO & $40(37)$ & $\operatorname{Ref}^{a}$ & & $101(55)$ & Ref & \\
\hline pN1a & $68(63)$ & $3.78(1.10$ to 12.97$)$ & 0.035 & $83(45)$ & 20.98 (2.78 to 158.30$)$ & 0.003 \\
\hline \multicolumn{7}{|l|}{ LN ratio } \\
\hline $\operatorname{Low}(\leq 0.4)$ & $39(57)$ & $1.15(0.23$ to 5.69$)$ & 0.866 & $50(60)$ & $10.14(1.18$ to 86.90$)$ & 0.034 \\
\hline High $(>0.4)$ & $29(43)$ & 40.80 (2.31 to 28.70$)$ & 0.001 & $33(40)$ & 40.80 (5.26 to 316.30$)$ & $<0.001$ \\
\hline \multicolumn{7}{|l|}{ LN size } \\
\hline Micrometastasis $(\leq 0.2 \mathrm{~cm})$ & $16(24)$ & $1.70(0.28$ to 10.15$)$ & 0.563 & $43(52)$ & 11.54 (1.35 to 98.79$)$ & 0.026 \\
\hline Macrometastasis $(>0.2 \mathrm{~cm})$ & $52(76)$ & 4.59 (1.32 to 15.98$)$ & 0.017 & $40(48)$ & 33.76 (4.35 to 262.21$)$ & $<0.001$ \\
\hline \multicolumn{7}{|l|}{ Patient group } \\
\hline Low & $14(20)$ & $0.00(-0.00$ to $\mathrm{NA})$ & 0.998 & $32(39)$ & $9.26(0.96$ to 89.01$)$ & 0.054 \\
\hline Intermediate & $27(40)$ & 2.97 (0.07 to 12.43$)$ & 0.136 & $29(35)$ & 14.46 (1.61 to 129.57$)$ & 0.017 \\
\hline High & $27(40)$ & 7.45 (2.07 to 26.79$)$ & 0.002 & $22(27)$ & 56.72 (7.16 to 449.11$)$ & $<0.001$ \\
\hline
\end{tabular}

$\mathrm{Cl}$, confidence interval; Ref, reference; NA, not available; $L N$ ratio, metastatic $L N s /$ removed LNs; Patient group according to the ratio and size of $L N$. apNO is a reference group to compare risk of recurrence in $L N$ ratio, $L N$ size, and patient group. 
analysis for patient group according to LN ratio and size showed poorer prognosis in pNO group than Low group (Table 3 and Fig. 3). One possible reason is the failure to detect clinically apparent LNs during operation. Alternatively, enlarged LNs caused by inflammatory change may have led surgeons to make no further efforts to find other suspicious metastatic LNs.

Of 184 patients in the prophylactic dissection group, 83 patients $(45 \%)$ were pN1. LN ratio and size were also significant risk factors in this group. The patient groups according to $\mathrm{LN}$ ratio and size were well correlated with prognosis $\left(\chi^{2}=43.9, \quad P<0.001\right)$ (Table 3 and Fig. 3).
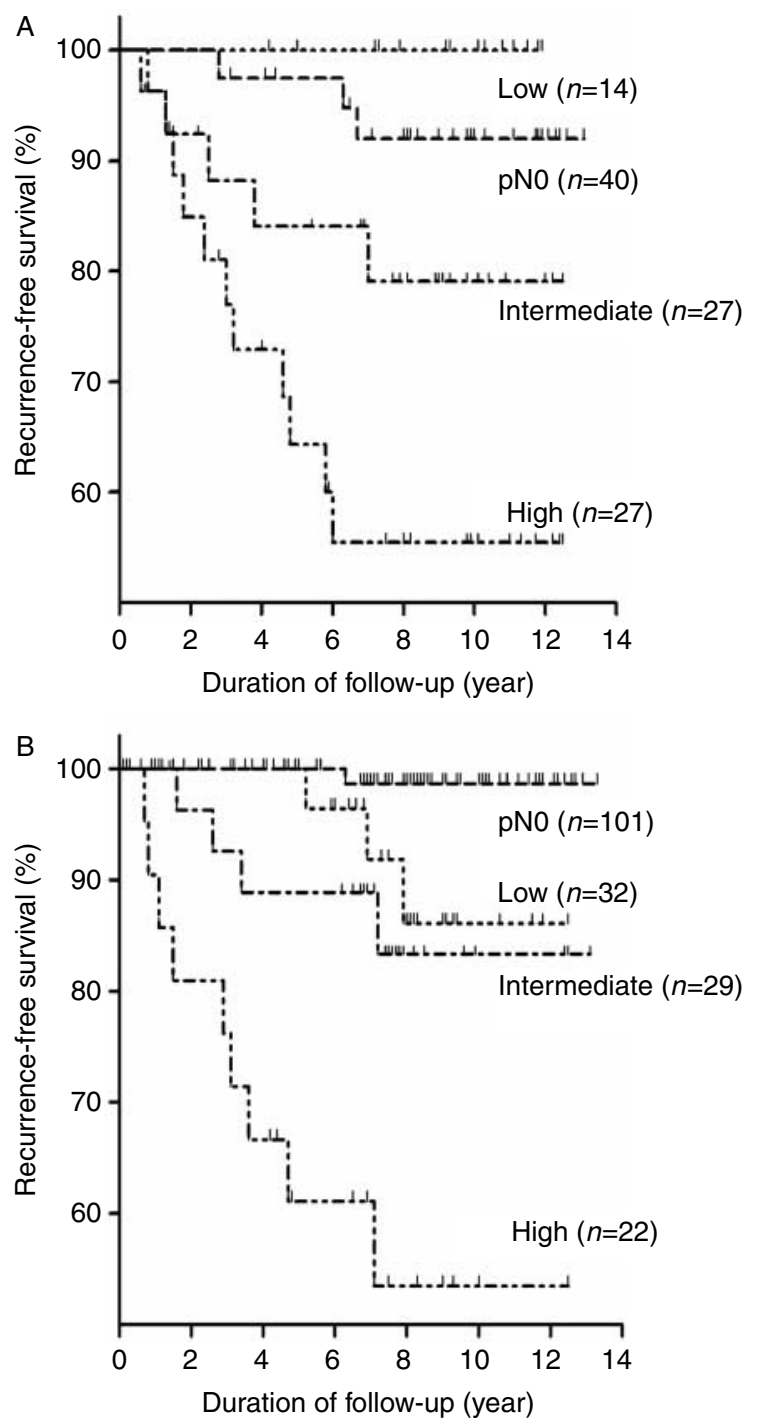

Figure 3 Recurrence-free survival according to the patient group classified by both LN ratio and size in each dissection group. (A) Kaplan-Meier curves for the therapeutic dissection group. These show poorer prognosis in the pNO group than in the Low group. (B) Kaplan-Meier curves for the prophylactic dissection group. These show close correlation of recurrence with patient group according to $\mathrm{LN}$ ratio and size (Log rank statistics $=43.9, P<0.001$ ).

\section{Discussion}

We showed the prognostic values of $\mathrm{LN}$ ratio and metastatic LN size on recurrence of PTC by comparing the risk of recurrences in $\mathrm{pNO}$ and $\mathrm{pN} 1 \mathrm{a}$ patients. To precisely assess the impact of metastatic pattern in central neck LN on prognosis, we only selected patients with primary tumor $>1 \mathrm{~cm}$ and without metastases to lateral neck or to distant organs. As only one diseasespecific mortality event occurred during the follow-up period of median 8 years, we could not evaluate the influence of prognostic factors on overall survival.

This study demonstrated that both high LN ratio and large LN size were positively associated with recurrence and LN ratio has a more prominent effect. As shown in Fig. 2, patient groups according to LN ratio and LN size were highly associated with prognosis. However, patients in group Low did not show a statistically significant increased risk of recurrence compared with those without pathologically proven metastatic LN. The others had poorer prognosis that was statistically significant and among them, group High, which had both high $\mathrm{LN}$ ratio and large metastatic LN, showed the lowest recurrence-free survival rate at $54.8 \%$.

Moreover, we found that older age at diagnosis and presence of microscopic perithyroidal extension did not have prognostic values on recurrence. Size of primary tumor, which was well known as an important prognostic factor for PTC, also showed no significant effect on recurrence in this study. Recurrence-free survival rate according to pathological $\mathrm{T}$ stage was $96.7 \%$ in $\mathrm{T} 1,76.5 \%$ in $\mathrm{T} 2$, and $84.6 \%$ in $\mathrm{T} 3$, and it seems that pathological $\mathrm{T}$ staging is not enough to predict prognosis (hazard ratio 1.53, 95\% CI 0.94 to $2.51, P=0.090)$. This is not consistent with the previous data $(1,10,11,12)$ and we think that patient selection bias might have led to this conclusion, as only those with tumor size $>1 \mathrm{~cm}$ were analyzed. Patients with tumor size $<1 \mathrm{~cm}$ showed overall excellent prognosis and it was not meaningful to analyze the impact of central neck LN metastases. We also excluded patients with gross invasion or resection margin involvement, which were associated with poorer prognosis (4).

Postoperative staging is important to assess the risk for disease recurrence, to make decisions on the frequency and intensity of follow-up and on postoperative adjunctive therapy. TNM classification is generally applied for differentiated thyroid cancer staging, but it was developed to predict risk for mortality and may not be appropriate for predicting recurrence in patients (4). TNM classification distinguishes central LN metastases from lateral neck metastases but does not take into consideration the extent of metastatic LN (1). The American Thyroid Association risk of recurrence system also did not use other characteristics of the LN metastases such as size or number of metastatic LNs (4). All patients with central neck metastases were treated 
in the same way, but we showed that their prognosis was extremely different according to $\mathrm{LN}$ ratio and to metastatic LN size. These results proved the need for new staging systems to predict accurate risk of recurrence of $\mathrm{pN} 1$ patients. This is consistent with the previous studies which showed that increased number of central LN metastases and LN size of larger than $3 \mathrm{~cm}$ were associated with poorer prognosis $(1,3,10,12,13$, 14, 17). Lang et al. (11) also revealed that higher metastatic central LN ratio (over 33.34\%) was an independent factor for short-term outcomes estimated by postablative stimulated serum thyroglobulin.

We believe that our findings may have important implications for clinicians. Patient's group considering metastatic LN ratio and size together would be a significant surrogate for determining RAI ablation dose and intensity of TSH suppression. Patients in group Low did not show any statistically significant increased risk of recurrence compared with pNO patients in multivariate analysis, and this suggests that lower RAI ablation dose could be applied or RAI ablation may be omitted in these selected lowrisk patients.

The surgical management of the central neck in patients without documented metastatic disease remains controversial and some suggested that prophylactic central neck dissection leads to an increase in $\mathrm{pN} 1$ disease that seems not to affect prognosis $(18,19,20)$. In this study, $184(63 \%)$ patients underwent prophylactic central neck dissection and there was no increase in recurrences in $\mathrm{cN} 1$ disease compared with $\mathrm{cNO}$ disease. We only included patients who underwent central neck dissection and could not compare the prognosis of prophylactic central neck dissection vs no prophylactic central neck dissection in $\mathrm{cNO}$ patients. About half of $\mathrm{cNO}$ patients who underwent prophylactic central neck dissection were $\mathrm{pN} 1$ disease and their prognosis was also well correlated with LN ratio, size, and patient's group. Our results suggest that discrete pathological LN staging after central neck dissection even prophylactic is an accurate predictor of recurrence. Further large studies are needed to evaluate this potential conflict.

Presence of microscopic ETE of tumor results in upstaging of $\mathrm{T} 1$ or $\mathrm{T} 2$ tumors to pathological $\mathrm{T} 3$ by current TNM staging system, but recent studies showed that microscopic ETE is of less significance than gross extension and patients' outcomes were not affected by microscopic ETE. Our findings along with previous data suggested that upstaging and administration of higher dose of RAI ablation due to microscopic ETE does not improve the prognosis $(21,22)$.

In conclusion, we found that recurrence-free survival in pN1a PTC patients was affected by the LN ratio and the size of metastatic LNs. Risk stratification for recurrence in pathological N1a patients according to LN ratio and size of metastatic LNs would give valuable information to clinicians.

\section{Declaration of interest}

The authors declare that there is no conflict of interest that could be perceived as prejudicing the impartiality of the research reported.

\section{Funding}

This study was supported by a Grant (2012-289) from the Asan Institute for Life Sciences, Seoul, Korea.

\section{References}

1 Leboulleux S, Rubino C, Baudin E, Caillou B, Hartl DM, Bidart JM, Travagli JP \& Schlumberger M. Prognostic factors for persistent or recurrent disease of papillary thyroid carcinoma with neck lymph node metastases and/or tumor extension beyond the thyroid capsule at initial diagnosis. Journal of Clinical Endocrinology and Metabolism 200590 5723-5729. (doi:10.1210/jc.20050285)

2 Teixeira G, Teixeira T, Gubert F, Chikota H \& Tufano R. The incidence of central neck micrometastatic disease in patients with papillary thyroid cancer staged preoperatively and intraoperatively as NO. Surgery 2011 150 1161-1167. (doi:10.1016/j.surg.2011. 09.019)

3 Ricarte-Filho J, Ganly I, Rivera M, Katabi N, Fu W, Shaha A, Tuttle RM, Fagin JA \& Ghossein R. Papillary thyroid carcinomas with cervical lymph node metastases can be stratified into clinically relevant prognostic categories using oncogenic BRAF, the number of nodal metastasis, and extra-nodal extension Thyroid 201222 575-584. (doi:10.1089/thy.2011.0431)

4 Cooper DS, Doherty GM, Haugen BR, Kloos RT, Lee SL, Mandel SJ, Mazzaferri EL, McIver B, Pacini F, Schlumberger M et al. Revised American Thyroid Association management guidelines for patients with thyroid nodules and differentiated thyroid cancer. Thyroid 200919 1167-1214. (doi:10.1089/thy.2009.0110)

5 Lee SR, Kim HO, Son BH, Shin JH \& Yoo CH. Prognostic significance of the metastatic lymph node ratio in patients with gastric cancer. World Journal of Surgery 201236 1096-1101. (doi:10.1007/s00268-012-1520-5)

6 Tausch C, Taucher S, Dubsky P, Seifert M, Reitsamer R, Kwasny W, Jakesz R, Fitzal F, Filipcic L, Fridrik M et al. Prognostic value of number of removed lymph nodes, number of involved lymph nodes, and lymph node ratio in 7502 breast cancer patients enrolled onto trials of the Austrian Breast and Colorectal Cancer Study Group (ABCSG). Annals of Surgical Oncology 201219 1808-1817. (doi:10.1245/s10434-011-2189-y)

7 Wong KP, Poon JT, Fan JK \& Law WL. Prognostic value of lymph node ratio in stage III colorectal cancer. Colorectal Disease 201113 1116-1122. (doi:10.1111/j.1463-1318.2010.02435.x)

8 Huvos AG, Hutter RV \& Berg JW. Significance of axillary macrometastases and micrometastases in mammary cancer. Annals of Surgery $1971 \mathbf{1 7 3}$ 44-46. (doi:10.1097/00000658197101000-00006)

9 Salhab M, Patani N \& Mokbel K. Sentinel lymph node micrometastasis in human breast cancer: an update. Surgical Oncology 201120 e195-e206. (doi:10.1016/j.suronc.2011.06. 006)

10 Lee YS, Lim YS, Lee JC, Wang SG, Kim IJ \& Lee BJ. Clinical implication of the number of central lymph node metastasis in papillary thyroid carcinoma: preliminary report. World Journal of Surgery 201034 2558-2563. (doi:10.1007/s00268010-0749-0)

11 Lang BH, Wong KP, Wan KY \& Lo CY. Significance of metastatic lymph node ratio on stimulated thyroglobulin levels in papillary thyroid carcinoma after prophylactic unilateral central neck dissection. Annals of Surgical Oncology 201219 1257-1263. (doi:10.1245/s10434-011-2105-5) 
12 Ito Y, Fukushima M, Kihara M, Takamura Y, Kobayashi K, Miya A \& Miyauchi A. Investigation of the prognosis of patients with papillary thyroid carcinoma by tumor size. Endocrine Journal 2012 59 457-464. (doi:10.1507/endocrj.EJ12-0013)

13 Sugitani I, Kasai N, Fujimoto Y \& Yanagisawa A. A novel classification system for patients with PTC: addition of the new variables of large $(3 \mathrm{~cm}$ or greater) nodal metastases and reclassification during the follow-up period. Surgery 2004135 139-148. (doi:10.1016/S0039-6060(03)00384-2)

14 Randolph G, Duh QY, Heller KS, Livolsi VA, Mandel SJ, Steward D, Tufano RP \& Tuttle RM. The prognostic significance of nodal metastases from papillary thyroid carcinoma can be stratified based on the size and number of metastatic lymph nodes, as well as the presence of extranodal extension ATA surgical affairs committee's taskforce on thyroid cancer nodal surgery. Thyroid $2012 \mathbf{1 1}$ 1144-1152. (doi:10.1089/thy.2012.0043)

15 Yim JH, Kim WB, Kim EY, Kim WG, Kim TY, Ryu JS, Gong G, Hong SJ \& Shong YK. The outcomes of first reoperation for locoregionally recurrent/persistent papillary thyroid carcinoma in patients who initially underwent total thyroidectomy and remnant ablation. Journal of Clinical Endocrinology and Metabolism 201196 2049-2056. (doi:10.1210/jc.2010-2298)

16 Sahin AA, Guray M \& Hunt KK. Identification and biologic significance of micrometastases in axillary lymph nodes in patients with invasive breast cancer. Archives of Pathology and Laboratory Medicine 2009133 869-878.

17 Xiao GZ \& Gao L. Central lymph node metastasis: is it a reliable indicator of lateral node involvement in papillary thyroid carcinoma? World Journal of Surgery 201034 237-241. (doi:10.1007/s00268-009-0347-1)

18 Carling T, Carty SE, Ciarleglio MM, Cooper DS, Doherty GM, Kim LT, Kloos RT, Mazzaferri EL, Peduzzi PN, Roman SA et al.
American Thyroid Association design and feasibility of a prospective randomized controlled trial of prophylactic central lymph node dissection for papillary thyroid carcinoma. Thyroid 201222 237-244. (doi:10.1089/thy.2011.0317)

19 Moreno MA, Edeiken-Monroe BS, Siegel ER, Sherman SI \& Clayman GL. In papillary thyroid cancer, preoperative central neck ultrasound detects only macroscopic surgical disease, but negative findings predict excellent long-term regional control and survival. Thyroid 201222 347-355. (doi:10.1089/thy.2011. 0121)

20 Popadich A, Levin O, Lee JC, Smooke-Praw S, Ro K, Fazel M, Arora A, Tolley NS, Palazzo F, Learoyd DL et al. A multicenter cohort study of total thyroidectomy and routine central lymph node dissection for cNO papillary thyroid cancer. Surgery 2011 150 1048-1057. (doi:10.1016/j.surg.2011.09.003)

21 Nixon IJ, Ganly I, Patel S, Palmer FL, Whitcher MM, Tuttle RM, Shaha AR \& Shah JP. The impact of microscopic extrathyroid extension on outcome in patients with clinical T1 and T2 welldifferentiated thyroid cancer. Surgery $2011 \quad 1501242-1249$. (doi:10.1016/j.surg.2011.09.007)

22 Ito Y, Tomoda C, Uruno T, Takamura Y, Miya A, Kobayashi K, Matsuzuka F, Kuma K \& Miyauchi A. Prognostic significance of extrathyroid extension of papillary thyroid carcinoma: massive but not minimal extension affects the relapse-free survival. World Journal of Surgery 200630 780-786. (doi:10.1007/s00268-0050270-z)

Received 23 August 2012

Revised version received 28 October 2012

Accepted 15 November 2012 